\title{
Sublinear growth of Information in DNA sequences
}

\author{
Giulia Menconi \\ Dipartimento di Matematica Applicata \\ and \\ C.I.S.S.C. Centro Interdisciplinare \\ per lo Studio dei Sistemi Complessi \\ Università di Pisa \\ Via Bonanno Pisano 25/b 56126 PISA - Italy \\ menconi@mail.dm.unipi.it \\ October 23, 2003
}

Running title: Sublinear Information in DNA

Keywords: Information Content, compression algorithm, DNA, repetitive sequences 


\begin{abstract}
We introduce a novel method to analyse complete genomes and recognise some distinctive features by means of an adaptive compression algorithm, which is not DNA-oriented. We study the Information Content as a function of the number of symbols encoded by the algorithm. Preliminar results are shown concerning regions having a sublinear type of information growth, which is strictly connected to the presence of highly repetitive subregions that might be supposed to have a regulatory function within the genome.
\end{abstract}

\title{
1 Introduction
}

We shall analyse the genome sequences from the point of view of data compression in order to exploit a linguistic analysis. As the context suggests, the genomes are interpreted as symbol sequences of finite length, drawn by an Information Source (the Nature) that remains mainly unknown and emits symbols taken from the alphabet of the four nucleotides $\{A, C, G, T\}$. Each genome identifies a living organism and we assume that it may be considered as the unique realisation produced by the Source relative to that organism.

We shall not give here a formal definition of Information Source. Intuitively, it is a device emitting a sequence of symbols $\ldots x_{1} x_{2} x_{3} \ldots$ where each $x_{i}$ is an element of a finite alphabet $\mathcal{A}$. The rigorous definition [1] lies on the notion of sequence space $\Omega_{\mathcal{A}}$, that is the space of one-sided infinite sequences (also called strings) $\omega=\left(\omega_{0}, \omega_{1}, \ldots\right)$ whose symbols are drawn from the alphabet. Even if an Information Source is rigorously defined as a stochastic process $\mathbb{X}=\left(\mathbb{X}_{n}\right)_{n \in \mathbb{N}}$ acting on a sequence space, we may consider the symbolic source $\Omega_{\mathcal{A}}$ as the subset of the sequence space containing all the realizations of the process $\mathbb{X}$. This shall motivate the use of the term Information Source when referring to a sequence space. We shall denote by $\mathcal{A}^{*}$ the set of finite symbolic sequences on the alphabet $\mathcal{A}$. If $s \in \mathcal{A}^{*}$ its length will be denoted by $|s|$.

DNA sequences are special quaternary symbol sequences. As only a small fraction of DNA nucleotides results in a viable organism, the sequences belonging to a living organism are expected to be nonrandom and have some constraints. Therefore, DNA sequences should be compressible, at least locally.

In our approach to symbol sequences, the crucial notion is the Information Content. Given a finite string $s$ in $\mathcal{A}^{*}$, the meaning of quantity of information $I(s)$ contained in $s$ has the following natural connotation:

$I(s)$ is the length of the smallest binary message from which you can reconstruct $s$. 
In his pioneering work, Shannon defined the quantity of information as a statistical notion using the tools of probability theory (2]). Thus in Shannon framework, the quantity of information which is contained in a string depends on its context. For example the string 'pane' contains a certain information when it is considered as a string coming from the English language. The same string 'pane' contains much less Shannon information when it is considered as a string coming from the Italian language because it is more frequent in the Italian language (in Italian it means "bread" and, of course, it is very frequent). Roughly speaking, the Shannon information of a string is the absolute value of the logarithm of the probability of its occurrence.

However, there are measures of information which depend intrinsically on the string and not on its probability within a given context. We will adopt this point of view. An example of these measures of information is the Algorithmic Information Content $(A I C)$. We will not formally define it (see [2] and [3] for rigorous definitions and properties). We limit ourselves to give an intuitive idea which is very close to the formal definition. We can consider a partial recursive function as a computer $C$ which takes a program $p$ (namely a binary string) as an input, performs some computations and gives a string $s=C(p)$, written in the given alphabet, as an output. The $A I C$ of a string $s$ is defined as the length of the shortest binary program $p$ which gives $s$ as its output, namely

$$
I_{A I C}(s, C)=\min \{|p|: C(p)=s\},
$$

where $|p|$ means the length in bit of the string which the program $p$ consists of. A theorem due to A. N. Kolmogorov (4]) implies that the information content $A I C$ of $s$ with respect to $C$ depends only on $s$ up to a fixed constant, therefore its asymptotic behaviour does not depend on the choice of $C$. The shortest program $p$ which outputs the string $s$ is a sort of optimal encoding of $s$. The information that is necessary to reconstruct the string is contained in the program. Unfortunately, this coding procedure cannot be performed by any algorithm. This is a very deep statement and, in some sense, it is equivalent to the Turing halting problem or to the Gödel incompleteness theorem. Then the Algorithmic Information Content is not computable by any algorithm.

Our method is focused on another measure: the information content of a finite string can also be defined by a lossless data compression algorithm $Z([3],[5])$. This turns out to be a Computable Information Content (CIC). In reference [6] quantitative relations among Shannon entropy of the source, the AIC and the CIC of sequences are provided.

The "classical" studies in compression algorithms answer the question about the compressibility of DNA with the additional advantage of using compression techniques to capture the properties of DNA. It is known that 
DNA sequences have two linguistic characteristic structures: reverse complements and approximate repeats. The reverse complement $\sigma^{c}$ of a sequence $\sigma$ is a sequence such that each symbol of $\sigma$ is replaced in $\sigma^{c}$ by its complement one. That is, reading the reverse complement of a subsequence from a single strand of DNA is the same as reading the corresponding complementary subsequence in the other strand. The approximate repeats are repeats that contain errors. Approximate repeats are due to the local variability that is a common feature within genomes.

There have been developed several special-purpose compression algorithms for DNA sequences (for instance, see [5], 7], 8], 9]). These algorithms are called DNA-oriented because they use the aforementioned charateristic structures of genomes together with a sort of statistical compression to achieve a compression ratio lower than two bits per symbol. This is a great improvement since the standard text compression algorithms such as compress or gzip cannot compress DNA sequences but only expand the file with more than two bits per symbol. The reason for text compression to fail on DNA sequences is that the regularities in genomes are much more subtler than in English texts, for which those algorithms have been designed.

Our analysis makes reference to a different approach. We aim at using the compression algorithm CASToRe, which has been created without any biological purpose and a priori linguistic knowledge, to understand whether there exist low information regions within a genome, whether they have a functional type in common, whether they are extended or have short length and what kind of growth the information content shows in those regions. Finally, as the algorithm CASToRe belongs to the class of algorithms that adaptively create a dictionary relative to a parsing of the input sequence, we shall study dictionaries after compression, in order to investigate the relations between patterns and biological functions.

\section{Computable Information Content}

Definition 1 (Compression Algorithm). A lossless data compression algorithm is any injective function $Z: \mathcal{A}^{*} \rightarrow\{0,1\}^{*}$.

Therefore, a compression algorithm is a reversible coding such that from the original string $s$ may be recovered from the encoded string $Z(s)$. Since the coded string contains all the information that is necessary to reconstruct and describe the structural features of the original string, we can consider the length of the coded string as an approximate measure of the quantity of information that is contained in the original string.

Definition 2 (Computable Information Content). The information content of a finite string $s \in: \mathcal{A}^{*}$ with respect to a compression algorithm $Z$ 
is defined as

$$
C I C_{Z}(s)=|Z(s)| .
$$

The CIC of a string $s$ is the length (in bit units) of the coded string $Z(s)$.

The advantage of using a compression algorithm lies in the fact that the information content $C I C_{Z}(s)$ is a computable function over the space of finite strings. For this reason we named it Computable Information Content.

Moreover, we define another quantity, the complexity of a finite sequence, providing an estimate for the rate of information content contained in it.

Definition 3 (Computable Complexity of a finite string). The complexity of $s$ with respect to $Z$ is the compression ratio

$$
K_{Z}(s)=\frac{I_{Z}(s)}{|s|} .
$$

Remark 1. Under suitable optimality assumptions on the compression algorithm $Z$, we can extend this definition to infinite symbolic sequences belonging to $\Omega_{\mathcal{A}}$ and asympotically obtain the Shannon entropy of the Information Source from which the sequence has been drawn $([10,11])$. The theoretical work has been extended also to trajectories coming from general dynamical systems and it is supported by application to several complex systems, as to turbulent or intermittent regimes $(12$, , 13, 14], 15], 16]) and to weakly chaotic dynamical systems $([17,[6])$.

\section{Dictionaries, words and phrases}

Let us describe the sort of linguistic analysis we shall perform on genetic sequences. We shall use the CIC method to extract the functional regions whose information content is low and its growth is sublinear. We aim at understanding whether those regions show peculiar features such as specific highly repeated patterns of nucleotides (they are usually called motifs). Finally, we shall scan other genomes, both coming from the same domain of life and from different domains, looking for the presence of low information regions and comparing the motifs to each other. These regions are called atypical, as surprisingly they are highly compressible in comparison with the other regions. The dictionaries of some atypical regions will be studied and related to some known biological functions (e.g. being a promoter region). Finally, a preliminar result on potential application of this method to gene finding will be introduced.

\subsection{The algorithm CASToRe}

We have created and implemented a particular compression algorithm we called CASToRe which is a modification of the Lempel-Ziv compression 
schemes $L Z 77$ and $L Z 78$ ([18, 19]) and it has been introduced and studied in references [12] and [17. Its theoretical advantages with respect to LZ78 showed that this algorithm is a sensitive measure of the Information content of low entropy sequences. This is the reason that motivates the choice of the acronym CASToRe to name the new algorithm: its meaning is Compression Algorithm, Sensitive To Regularity. As it has been proved in 17, the Information content $I_{Z}$ of a constant sequence $s^{n}$, originally with length $n$, is $\Psi(n)=4+2 \log (n+1)[\log (\log (n+1))-1]$, if the algorithm $Z$ is CASToRe. The theory predicts that the best possible information content for a constant sequence of length $n$ is $A I C\left(s^{n}\right)=\log (n)+$ constant. It may be shown that the algorithm $L Z 78$ encodes a constant $n$-digits long sequence to a string with length about const $+n^{\frac{1}{2}}$ bits; so, we cannot expect that $L Z 78$ is able to distinguish a sequence whose information content grows like $n^{\alpha}$ $\left(\alpha<\frac{1}{2}\right)$ from a constant or periodic string. Furthermore, the running time of CASToRe is also sensibly shorter than that of $L Z 77$ (with infinite window), then any implementation is more efficient. These are the main reasons that motivate the choice of using CASToRe also for numerical experiments.

Now we briefly describe the internal running of CASToRe.

As the Ziv-Lempel schemes, the algorithm CASToRe is based on an adaptive dictionary (20]). One of the basic differences in the coding procedure is that the algorithm $L Z 77$ splits the input strings in overlapping phrases, while the algorithm CASToRe (as well as the algorithm LZ78) parses the input string in non-overlapping phrases. Moreover, CASToRe differs from $L Z 78$ because the new phrase is a pair of two already parsed phrases, while $L Z 78$ couples one already parsed phrase and one symbol from the alphabet.

At the beginning of encoding procedure, the dictionary contains only the alphabet. In order to explain the main rules of the encoding, let us consider a step $h$ within the encoding process, when the dictionary already contains $h$ phrases $\left\{e_{1}, \ldots, e_{h}\right\}$.

The new phrase is defined as a pair (prefix pointer,suffix pointer). The two pointers are referred to two (not necessarily different) phrases $\rho_{p}$ and $\rho_{s}$ chosen among the ones contained in the current dictionary as follows. First, the algorithm reads the input stream starting from the current position of the front end, looking for the longest phrase $\rho_{p}$ matching the stream. Then, the algorithm looks for the longest phrase $\rho_{s}$ such that the joint word $\rho_{p}+\rho_{s}$ matches the stream. The new phrase $e_{h+1}$ that will be added to the dictionary is then $e_{h+1}=\rho_{p}+\rho_{s}$.

The output file contains an ordered sequence of the binary encoding of the pairs $\left(i_{p}, i_{s}\right)$ such that $i_{p}$ and $i_{s}$ are the dictionary index numbers corresponding to the prefix word $\rho_{p}$ and to the suffix word $\rho_{s}$, respectively. The pair $\left(i_{p}, i_{s}\right)$ is referred to the new encoded phrase $e_{h+1}$ and has its own index number $i_{h+1}$. 


\subsubsection{Example}

The following example shows how the algorithm CASToRe encodes the input stream

$$
\omega=(a b c a b a b c c a b b \ldots) .
$$

Let the source alphabet be $\mathcal{A}=\{a, b, c\}$.

The output file corresponds to the binary encoding of the following pairs contained in the second column. The first column is the dictionary index number of the encoded phrase in the dictionary which is showed in the same line, second column. For an easier reading, we add a third column which shows each encoded phrase in the original stream $\omega$, but which is not contained in the output file:

First, the alphabet is loaded

$\begin{array}{lll}1 & \left(0,{ }^{\prime} a^{\prime}\right) & {[a]} \\ 2 & \left(0,{ }^{\prime} b^{\prime}\right) & {[b]} \\ 3 & \left(0,{ }^{\prime} c^{\prime}\right) & {[c]} \\ & \text { Then, the encoding procedure starts } & \\ 4 & (1,2) & {[a b]} \\ 5 & (3,4) & {[c a b]} \\ 6 & (4,3) & {[a b c]} \\ 7 & (5,3) & {[c a b c]}\end{array}$

and so on.

\subsection{Reading the dictionary}

The dictionary built by the algorithm CASToRe is an ordered collection of phrases, that is, of pairs of words. Thus, a phrase is composed by a prefixword and a suffix-word. By construction, phrases are different from each other, since the algorithm exploits a parsing on the input string. Furthermore, each phrase may become a word, if it appears as prefix or suffix of other phrases in the following dictionary.

In the following, we shall look at the most frequent words, at the longest phrases and in some cases we shall compare the results to the same analysis performed by means of the algorithm $L Z 77$ and exploited in collaboration with a group of physicists from the University of Rome (see their previous work 21] by V. Loreto et al. for details on the methodology). We shall show that recurrent subsequences occur especially along the regions with lowest information content. Notice that we refer to exact repeats.

We shall distinguish among recurrent subsequences either motifs or patterns. A motif is a recurrent word in the dictionary, whereas a pattern is a recurrent subsequence that does not match any word of the dictionary, but is contained in some of them. If a motif is found, we shall follow its descent, that is the set of phrases whose the motif is either a prefix or a suffix or 
both. Moreover, we shall search for the motif to be a sliding pattern, in the sense that it is contained in other phrases without being their prefix nor their suffix. Furthermore, if only a sliding pattern is to be found, then we shall recover its root, that is the longest word of the dictionary matching part of the pattern.

\section{The Information Content of DNA sequences}

We have analysed the computable complexity of 12 complete genomes $^{1}$ of some Archaea, Bacteria and Eukaryotes, together with chromosomes II and IV of Arabidopsis thaliana. The complete list is shown on the following Table 1

In order to take into account the biological functional constraints actually existing among the bases within the genome and to highlight new features of coding and noncoding regions, we have exploited a fragment analysis.

Definition 4. We say that any exon, intron or intergenic region is a functional fragment of the genome sequence, following the prediction as it has been identified via biological databases and statistical tools ([22]).

Notation. In prokariotic genomes there are two functional types, therefore we shall denote by Coding_\# and Inter_\# the coding and the noncoding fragments, respectively, where \# is an index to order fragments. In eukaryotic genomes there are three different types of regions: we shall denote by Exon_\# the coding fragments and by Intron_\# and Inter_\# the noncoding intragenic fragments and the noncoding integenic fragments, respectively.

Thus, we shall consider the Computable Complexity $K(f)$ of each fragment and study the Information Content growth $C I C(f)$ within a fragment.

First, we have considered how the Information Content varies along some complete DNA sequences: that is, we have studied the behaviour of the CIC of a genome as a function of the number of encoded symbols. As a result, we remark that the function $C I C\left(\sigma_{n}\right)$ grows linearly for all the complete genomes $\sigma$ we have analysed and the asymptotic slope is the value of their Computable complexity $K(\sigma)$ :

$$
C I C\left(\sigma_{n}\right) \sim K(\sigma) \cdot n,
$$

where $\sigma_{n}$ indicates the first $n$ bases in the complete genome $\sigma$. However, we can enhance some regions of the genome and we will see that the $C I C$-line is locally no more straight. This characteristic feature is shared by all the genomes we have analysed, both Prokaryotes and Eukaryotes and confirms the intuitive idea that the Information Content growth should be slower in the parts of the genome where some regularity prevails.

\footnotetext{
${ }^{1}$ The genomes have been downloaded by means of the GenBank sequence libraries http://www.ncbi.nlm.nih.gov/Genbank/index.html
} 


\begin{tabular}{|c|c|c|}
\hline Genome & CSS & $H_{1}$ \\
\hline \hline Methanococcus jannaschii & 1.794 & 1.887 \\
\hline Archeoglobus fulgidus & 1.909 & 1.987 \\
\hline Methanobacterium thermoautrophicum & 1.907 & 1.986 \\
\hline Pyrococcus abyssi & 1.901 & 1.979 \\
\hline \hline Aquifex aeolicus & 1.883 & 1.976 \\
\hline Escherichia coli & 1.893 & 1.987 \\
\hline Bacillus subtilis & 1.870 & 1.975 \\
\hline Haemophylus influenzae & 1.866 & 1.947 \\
\hline Mycoplasma genitalium & 1.848 & 1.959 \\
\hline \hline Thermotoga maritima & 1.893 & 1.984 \\
\hline Arabidopsis thaliana (chr. II and IV) & 1.892 & 1.938 \\
\hline Saccharomyces cerevisiae & 1.889 & 1.949 \\
\hline Caenorhabditis elegans & 1.777 & 1.936 \\
\hline
\end{tabular}

Table 1: complete genomes. Comparison CSS vs. $H_{1}$.

For instance, see the results about the genome of Archaeoglobus fulgidus (Prokaryote) which are pictured on figure1. For the sake of brevity, we shall not show analogous pictures coming from other genomes.

For what concerns the values of computable complexity $K$ for the complete genomes we have analysed, the results are shown on Table1. We have indicated the complexity $K$ as $C S S$, meaning complexity as a single string, to distinguish it from the fragment complexity, which is the value of the computable complexity of the functional fragments within the complete genome and which will be denoted by $F C$ in the following. The final column in Table 1 shows the first order entropy $H_{1}$ of the sequence. If $p_{A}, p_{C}, p_{G}, p_{T}$ are the nucleotide frequencies over a genome $\sigma$ (the frequency is calculated as the number of occurrences of a specific nucleotide over the total number of nucleotides), then the first order entropy is $H_{1}=\sum_{i=A, C, G, T} p_{i} \log p_{i}$. We recall that, when the symbols are drawn uniformly at random from the source and all the positions in the sequence are independent from each other, an optimal coding procedure will devote $\log _{2}(\# \mathcal{A})$ bits per symbol to represent each character $(23])$, where $\# \mathcal{A}$ is the number of symbols in the alphabet $\mathcal{A}$. In this case the asymptotically maximal complexity equals the $H_{1}$ value for those values of nucleotide frequencies. For quaternary sequences, like the genomes, this maximal mean first order entropy is 2 bits per symbol. Since the $H_{1}$ value represents a quantity of information of a single string which is dependent on the probability measure on the space of sequences, at first sight the genomes cannot be considered randomly distributed (from a statistical point of view), because for all of them the $H_{1}$ values are different from 2 bits per symbol. First, we notice that the values of the complexity 
$C S S$ are significantly different from 2 and lower than the $H_{1}$ entropy values. Again, this is in complete agreement with the fact that the randomness of the genomes has strong constraints. It is also possible to clearly recognise that some genomes have very low computable complexity (smaller than 1.90 bits per symbol), which means that their internal structure presents mid-range and long-range correlations.

The compression of complete genomes does not satisfy the quest for local structures along a genome. The presence of local nonlinearities in the Information Content function for complete genomes suggests the existence of specific functional fragments whose Information Content function grows sublinearly. We recall that we named those regions atypical. Consequently, we shall investigate in this direction by means of the fragment analysis.

\subsection{A sublinearity index}

In order to identify the regions where the growth of the function $C I C\left(\sigma_{n}\right)$ is sublinear, we define a sublinearity index, that allows us to determine whether a functional region is atypical.

In the following, $\sigma$ shall denote any fragment within a genome. The sublinearity index may be defined by means of any adaptive compression algorithm $Z$, although the experimental results are referred to the algorithm CASToRe.

Let $N=|\sigma|$ be the length of the input sequence $\sigma$. Let $\mathcal{P}(\sigma, Z)$ be the parsing of $\sigma$ with respect to the algorithm $Z: \mathcal{P}(\sigma, Z)=\left\{\phi_{1}, \phi_{2}, \ldots, \phi_{t}\right\}$. Therefore, the input string $\sigma$ is the ordered juxtaposition of phrases $\phi_{j}$ 's. We use the symbol $n_{k}$ to indicate the current total number of encoded symbols up to step $k$ of the encoding procedure: $n_{k}=\Sigma_{j=1}^{k}\left|\phi_{j}\right|$. Due to the fact that $\left|\phi_{k}\right|=n_{k}-n_{k-1}$, we say that $n_{k}$ is the parsing index corresponding to the phrase $\phi_{k}$. The Information Content after $k$ steps is then the quantity $I\left(n_{k}\right)=\Sigma_{j=1}^{k} I\left(\phi_{j}\right)$. Obviously, it holds that $n_{t}=\Sigma_{j=1}^{t}\left|\phi_{j}\right|=N$ and $I(\sigma)=$ $I(N)=\Sigma_{j=1}^{t} I\left(\phi_{j}\right)$. Since the encoding procedure might be not precise in the early steps as well as in the final steps, we fix two bounds defining the restriction of the potential integer value $n_{j}$. Let $T_{i n f}=20 \%|\sigma|$ be the lower bound and $T_{\text {sup }}=90 \%|\sigma|$ be the upper bound. The choice of the bounds will be such that there exist two parsing indexes $n_{\text {inf }}$ and $n_{\text {sup }}$ such that $T_{\text {inf }} \leq n_{\text {inf }}<n_{\text {sup }} \leq T_{\text {sup }}$. Moreover, since the algorithm $Z$ requires that the input sequence is sufficiently long to make the compression reliable and efficient, we shall not analyse sequences whose length $N$ is lower than 200 symbols. Thus, for the set $\left\{n_{j} \| j=1, \ldots, t\right\}$ coming from the parsing of $\sigma$ via the algorithm $Z$, we define the domain $\mathcal{D}=\left\{n_{k} \| n_{\text {inf }} \leq n_{k} \leq\right.$ $\left.n_{\text {sup }}, n_{t} \geq 200\right\}$.

\section{Definition 5 (Sublinearity index of a finite symbol sequence).}


Let $q_{\min }, q_{\max }$ and $q_{Z}(\sigma)$ be defined as follows:

$$
\begin{aligned}
q_{\text {min }} & =\min _{n_{k} \in \mathcal{D}}\left\{\frac{I\left(n_{k}\right)}{n_{k}}\right\}, \\
q_{\max } & =\max _{n_{k} \in \mathcal{D}}\left\{\frac{I\left(n_{k}\right)}{n_{k}}\right\}
\end{aligned}
$$

and

$$
q_{Z}(\sigma)=\frac{q_{\min }}{q_{\max }} .
$$

The sublinearity index $\mathcal{G}_{Z}(\sigma)$ of the input sequence $\sigma$ with respect to the parsing defined via the algorithm $Z$ is the quantity

$$
\mathcal{G}_{Z}(\sigma)=\frac{\log \left(q_{Z}(\sigma)\right)}{\log \left(\frac{n_{\text {sup }}}{n_{\text {inf }}}\right)}+1
$$

The definition of this index $\mathcal{G}_{Z}$ deserves some comments. Its main characteristic is that it allows a criterion to identify atypical regions to be established.

First of all, it is known that the behaviour of the Information Content of a finite sequence $\sigma$ is an increasing function $I\left(\sigma^{n}\right)$ that grows at most linearly with the number $n$ of encoded symbols. Therefore, the indexes $q_{\min }$ and $q_{\max }$ can be easily calculated by:

$$
q_{\min }=\frac{I\left(n_{\text {sup }}\right)}{n_{\text {sup }}} \text { and } q_{\max }=\frac{I\left(n_{\text {inf }}\right)}{n_{\text {inf }}} .
$$

Hence, it is straightforward that the value of the sublinearity index is

$$
\mathcal{G}_{Z}(\sigma)=\frac{\log \left(I\left(n_{\text {sup }}\right)\right)-\log \left(I\left(n_{\text {inf }}\right)\right)}{\log \left(n_{\text {sup }}\right)-\log \left(n_{\text {inf }}\right)} .
$$

We notice that the fragment we have analysed are not periodic, otherwise the phrases found in the parsing by the algorithm CASToRe would definitely show length doubling, which is absent in the dictionaries of all the fragments. Furthermore, the Information Content growth of any functional fragment $\sigma$ can not be a logarithmic function $\Psi(n)$ (see Section 3.1), but we might assume that it can be read $(\forall 1 \leq n \leq|\sigma|)$ as

(5) $C I C\left(\sigma_{n}\right)=\mathcal{O}\left(C n^{\gamma}\right)$, with exponent $0<\gamma \leq 1$ and constant $C>0$.

Note that this formula is relative to a finite sequence, therefore the writing $\mathcal{O}\left(\mathrm{Cn}^{\gamma}\right.$ ) is not referring to an asympotic behaviour (as $n \leq|\sigma|$ ), but it means that the integer function $C I C\left(\sigma_{n}\right)$ is fitted by a function whose dominant term is a power law with exponent smaller than 1 . Since we have excluded any pure periodicity, hypothesis (5) is doubtless plausible. 


\begin{tabular}{|c|c|c|c|}
\hline Genome & Sequence & value of $\mathcal{G}_{Z}$ & fit-value of $\gamma$ \\
\hline \hline Archaeoglobus fulgidus & Coding_685495 & 0.965 & 1.000 \\
\hline Archaeoglobus fulgidus & Inter_1143603 & 0.949 & 0.949 \\
\hline Archaeoglobus fulgidus & Inter_393196 & 0.832 & 0.831 \\
\hline \hline Escherichia coli & Inter_2302612 & 0.768 & 0.747 \\
\hline Escherichia coli & Inter_4293752 & 0.728 & 0.730 \\
\hline Escherichia coli & Coding_91419 & 0.986 & 0.986 \\
\hline \hline Arabidopsis thaliana & Exon_23950656 & 0.614 & 0.585 \\
\hline Arabidopsis thaliana & Intron_5063613 & 0.767 & 0.738 \\
\hline Arabidopsis thaliana & Inter_19660110 & 0.887 & 0.886 \\
\hline
\end{tabular}

Table 2: reliability of the sublinearity index $\mathcal{G}_{Z}$ in the case of several functional regions from different genomes.

The two following main points are definitely true. First, a sublinear growth of Information Content is an indicator of the presence of some regularity in the input sequence and this is much more evident when the index $\mathcal{G}_{Z}$ is significantly smaller than 1 . Second, small values of the index $\mathcal{G}_{Z}$ may correspond to different sublinear information growths - also other than power-law-like - that consequently might be a signal of different underlying dynamics generating the symbol sequences.

In the following Lemma, the sublinearity index $\mathcal{G}_{Z}$ in the case of Information Content growing exactly as a power law is evaluated.

Lemma 1. If $C I C\left(n_{k}\right)=C n_{k} \gamma$ with $0<\gamma \leq 1$, then $\mathcal{G}_{z}=\gamma$.

Proof. Consider the formula (4). In this case, it holds that

$$
\mathcal{G}_{Z}=\frac{\log (C)+\alpha \log \left(n_{\text {sup }}\right)-\log (C)-\alpha \log \left(n_{\text {inf }}\right)}{\log \left(n_{\text {sup }}\right)-\log \left(n_{\text {inf }}\right)} .
$$

Therefore, the conclusion is straightforward.

Thus, according to formula (5), the sublinearity index $\mathcal{G}_{Z}$ is a reliable quantity that allows the degree of sublinearity of the information content growth to be estimated. In order to evaluate the precision of the index $\mathcal{G}_{Z}$ with respect to the true actual exponent $\gamma$, we have compared the values of $\mathcal{G}_{z}$ with the values of $\gamma$ as they are given by a numerical fit on the integer function $I(n)$. The results are definitely satisfactory. Some examples are shown on Table 2 and are referred to several fragments from the genomes of Archaeoglobus fulgidus, Escherichia coli and Arabidopsis thaliana.

The following definition will be used to extract the atypical functional regions. The threshold has been fixed according to the empirical principle that the kind of growth $n^{\gamma}$ where $\gamma$ lies in $[0.9,1]$ is, on a general basis, 
equivalent to a linear growth, due to the finiteness of the sequences under analysis.

Definition 6 (Atypical region). An atypical region within a genome is any functional region whose sublinearity index $\mathcal{G}_{Z}$ is smaller than 0.9 .

The connection between sublinearity index and fragment complexity is not precise, even if in the extreme cases where both values are either high or low a sort of clusters are detected. For instance, Figure 3 illustrates what the relation is between the sublinearity index (horizontal axis) and the fragment complexity (vertical axis) in the case of the genome of Archaeoglobus fulgidus. Atypical regions are indicated by means of a vertical line that represents the threshold for the sublinearity index as introduced in Definition 6 . It is clear that, both in the case of coding regions (depicted by a cross) and of noncoding regions (depicted by a diamond), the higher the fragment complexity is, the higher the sublinearity index is. Furthermore, the detection of atypical regions with high fragment complexity suggests that the sublinearity index may be more meaningful in identifying regularity of sequences than the fragment complexity.

\section{Experimental results}

In the following, we shall introduce some preliminar examples of application of the CIC method. Ww shall analyse the dictionary of some long atypical regions within the genomes of Archaeoglobus fulgidus, Methanococcus jannaschii and Arabidopsis thaliana. We shall discover peculiar properties and propose some biological motivations to those features. This part of the work has been developed in collaboration to the Animal Biology and Genetics Department of the University of Florence.

\subsection{Archaeoglobus fulgidus}

Archaeoglobus fulgidus is a sulphur-metabolizing anaerobic organism. It belongs to the Archaeoglobales, archaeal sulfate reducers unrelated to other sulfate reducers. They grow at extremely high temperatures. Archaeoglobus species causes corrosion of iron and steel in oil and gas processing systems by the production of iron sulphide. This organism has one circular chromosome.

Looking at Figure 4 we have extracted two regions: one atypical region, which is noncoding, and two non-atypical regions, one coding and one noncoding. This choice is aimed at comparing the dictionaries of regions with sublinear grwoth of information to the dictionaries of regions with linear growth of information.

The exemplified regions are 
- Coding_685495: non-atypical region, length $L=2300$ bp, sublinearity index $\mathcal{G}_{Z}=0.965$, fragment complexity $K=2.108$;

- Inter_1143603: non-atypical region, length $L=2219 \mathrm{bp}$, sublinearity index $\mathcal{G}_{Z}=0.949$, fragment complexity $K=2.117$;

- Inter_393196: atypical region, length $L=2629 \mathrm{bp}$, sublinearity index $\mathcal{G}_{Z}=0.832$, fragment complexity $K=1.494$.

We start analysing the non-typical regions. First, we have plotted the length of the phrases in the dictionary together with their position in the input sequence (see Figure $5(a)$ and $(b)$ ). In both non-atypical regions, the phrases are short and the maximal length is $11 \mathrm{bp}$. The Gaussian distribution of phrase length confirms that these regions are not regular, but highly variable (see Figure $5(c)$ and $(d)$ ). The extent of the dictionary is great in both nonatypical regions: 415 phrases in the dictionary of region Coding_685495 and 393 phrases in the dictionary of region Inter_1143603.

However, in the case of region Coding_685495, the algorithm CASToRe recognised 31 codons as phrases that are also used as prefix or suffix words quite frequently. Table 3 illustrates the details of this feature that has been found only in coding regions; in fact, in non-atypical noncoding regions the codons that are recognised as phrases are always a few.

Conversely, the dictionary relative to fragment Inter_393196, which is atypical noncoding, shows completely different characteristics. First of all, the dictionary contains 349 phrases. Moreover, Figure 6 (a) shows that in this sequence there should be recurrences of similar patterns, because of the several long phrases (that is, longer than $25 \mathrm{bp}$ ) that are spread along the whole sequence. Another feature, which will be paradigmatic of atypical regions, is the anomalous (non-Gaussian) tail in the distribution of phrase length (see Figure 6 (b)). The distribution is no longer peaked at only one value, but there is a significant occurrence of long words that could not be found in non-atypical regions and is consistent with the presence of regularity within any atypical region.

According to the dictionary obtained by means of algorithm CASToRe, there is a dominant motif $\mathcal{M}$ of length $25 \mathrm{bp}$ (phrase nr. 109 in the dictionary), that is also used 9 times as a prefix and 3 times as a suffix. Table 4 illustrates what the dominant motif $\mathcal{M}$ and its descent are. We recall that the descent of a phrase $\phi$ is the set of other phrases in the dictionary such that $\phi$ is either their prefix or suffix or both.

The presence of a dominant motif partially motivates the many oscillations in the $C I C$ growth, as depicted in Figure 2 Furthermore, a complete explanation lays on the fact that the motif $\mathcal{M}$ is also a sliding pattern in many other phrases (see Table 5). This is an irrefutable evidence of the fact that this atypical region shows a variable periodicity represented by the 


\begin{tabular}{|c|c|c||c|c|c|}
\hline Codon & \# prefix & \# suffix & Codon & \# prefix & \# suffix \\
\hline \hline AAA & 10 & 4 & CTG & 8 & 7 \\
\hline AAG & 1 & 8 & GCA & 3 & 2 \\
\hline AAT & 4 & 1 & GCC & 8 & 6 \\
\hline ACA & 10 & 4 & GCT & 5 & 3 \\
\hline ACC & 4 & 7 & GGA & 2 & 4 \\
\hline ACG & 4 & 1 & GGT & 2 & 2 \\
\hline ACT & 7 & 6 & TAA & 5 & 8 \\
\hline ATG & 3 & 0 & TAG & 0 & 1 \\
\hline ATT & 4 & 8 & TAT & 2 & 4 \\
\hline CAA & 14 & 10 & TCA & 6 & 6 \\
\hline CAG & 3 & 7 & TCC & 8 & 2 \\
\hline CAT & 0 & 0 & TCT & 7 & 5 \\
\hline CCG & 3 & 1 & TGT & 2 & 6 \\
\hline CCT & 2 & 3 & TTA & 5 & 7 \\
\hline CGG & 0 & 0 & TTG & 0 & 4 \\
\hline CGT & 5 & 7 & & & \\
\hline
\end{tabular}

Table 3: 31 different codons have been recognised as phrases in the parsing by the algorithm CASToRe, in region Coding_685495 of the genome of Archaeoglobus fulgidus. Some of them have been also used as prefix or suffix of other phrases. Columns named \# prefix and \# suffix indicate how many times the phrase has been used as a prefix or suffix.

\author{
$\mathcal{M}=$ AATCCCATTTTGGTCTGATTTCAAC \\ Descent of $\mathcal{M}$ : \\ AATCCCATTTTGGTCTGATTTCAACACA \\ AATCCCATTTTGGTCTGATTTCAACAG \\ AATCCCATTTTGGTCTGATTTCAACCAA \\ AATCCCATTTTGGTCTGATTTCAACCT \\ AATCCCATTTTGGTCTGATTTCAACGA \\ AATCCCATTTTGGTCTGATTTCAACGT \\ AATCCCATTTTGGTCTGATTTCAACTATTT \\ AATCCCATTTTGGTCTGATTTCAACTT \\ AATCCCATTTTGGTCTGATTTCAACTTTC \\ CССТTТСАATCCCATTTTGGTCTGATTTCAAC \\ CTTTCAATCCCATTTTGGTCTGATTTCAAC \\ TTTCAATCCCATTTTGGTCTGATTTCAAC
}

Table 4: dominant motif $\mathcal{M}$ and its descent in atypical region Inter_393196 of the genome of Archaeoglobus fulgidus. 


$\mathcal{M}=$ AATCCCATTTTGGTCTGATTTCAAC
Motif as a sling pattern in:
TTCAATCCCATTTGGTCTGATTCAAC
AATCCCATTTTGGTCTGATTTCAACGAAG
AATCCCATTTTGGTCTGATTTCAACCTCC
AATCCCATTTTGGTCTGATTTCAACTATTT
CTTTCAATCCCATTTTGGTCTGATTTCAAC
CCTTTCAATCCCATTTTGGTCTGATTTCAAC
TCTTTCAATCCCATTTTGGTCTGATTTCAAC
TTCAATCCCATTTTGGTCTGATTTCAACTCC
TTTCAATCCCATTTTGGTCTGATTTCAACCTT
CGCTTTCAATCCCATTTTGGTCTGATTTCAAC
AATCCCATTTTGGTCTGATTTCAACGAGGCGT
CCCTTTCAATCCCATTTTGGTCTGATTTCAAC
CTCCTTTCAATCCCATTTTGGTCTGATTTCAAC
CCTTTCAATCCCATTTTGGTCTGATTTCAACTA
ACTTTCAATCCCATTTTGGTCTGATTTCAACAG
TTTCAATCCCATTTTGGTCTGATTTCAACTTTA
CTTTCAATCCCATTTTGGTCTGATTTCAACATC
GTCTCTTTCAATCCCATTTTGGTCTGATTTCAAC
CACGCTTTCAATCCCATTTTGGTCTGATTTCAAC
ACCCCTTTCAATCCCATTTTGGTCTGATTTCAAC

Table 5: phrases where the motif $\mathcal{M}$ is a sliding pattern. The motif is written bold typed.

recurrence of the motif $\mathcal{M}$ sometimes slightly modified, as in the case of approximate repeats.

Even if the biological usefulness of the motif $\mathcal{M}$ is still unknown, another hint to its peculiarity is provided by the compression of region Inter_393196 by means of algorithm $L Z 77$. The motif $\mathcal{M}$ is a motif also in the dictionary extracted by $L Z 77$. Therefore, the idea that this motif should have a precise biological meaning is even more convincing. Furthermore, this example suggests that also approximate repeats generated by insertions may be identified via $C I C$ method.

\subsection{Methanococcus jannaschii}

Methanococcus jannaschii is a thermophilic $\left(48-94^{\circ} \mathrm{C}\right)$, strict anaerobic Archaebacterium living at pressures of over 200 atmospheres. It is an autotroph which gets its energy from hydrogen and carbon dioxide producing methane and it is capable of nitrogen fixation. Morphologically, it is characterized by having two bundles of flagella at the same cellular pole. The genome 
of Methanococcus jannaschii consists of the main circular chromosome and two circular extrachromosomal elements (ECE), one large and one small. We have analysed only the main chromosome.

In this genome we shall show one atypical region, whose sublinearity index is particularly low and having approximately the same extent as the other regions that have been already analysed. However, as it is shown on Figure 7 this genome presents many other long atypical regions, that will be studied in future work.

The atypical region we have analysed is

- Inter_236189: atypical region, length $L=2112 b p$, sublinearity index $\mathcal{G}_{Z}=0.707$, fragment complexity $K=1.405$.

The behaviour of the information content in atypical region Inter_236189 is twofold: until the first 1500 base pairs have been encoded, the growth is almost logarithmic, while in the final part the $C I C$ increase is faster (see Figure (8). Therefore, the first part of the sequence should be more regular than the second one.

This aspect is well-represented in graph $(a)$ of Figure 9. The presence of longer and longer phrases before $1500 \mathrm{bp}$ have been compressed is an evidence for the existence of highly repetitive subsequences in the first half, whereas in the second half of the input sequence Inter_236189 the previous regularity is broken and only brief repetitions can be found. Consequently, the extent of the dictionary is low: there are only 264 phrases.

As in the case of the analysed atypical region of genome of Archaeoglobus fulgidus, the distribution of phrase length has an anomalous non-Gaussian tail that comprehends also a phrase that is $134 \mathrm{bp}$ long (Figure $9(b)$ ).

For what concerns the analysis of recurrent phrases in the dictionary, it holds that only phrases that are shorter than $10 \mathrm{bp}$ are used more than three times as prefix word or suffix word. As it is shown in Table 6. the phrases longer than 20 bp (that correspond to the high "spikes" of Figure $9(a)$ ) do not allow a dominant motif to be determined in such a definite way as in the case of atypical region Inter_393196 of Archaeoglobus fulgidus genome. The increasingly longer phrases that have been detected in graph 9 (a) are not generated by coupling the prefix word to itself (as it would have been if there were a precise periodicity), but prefix and suffix words were different from each other and neither they are subsequent. Again, the longest phrases coincides with the longest ones found by means of the algorithm $L Z 77$.

However, the main point of distinction of this atypical region is that all long phrases are rich in $\mathrm{T}^{n} \mathrm{~A}^{m}$-patterns. This fact, together with the positive homology response classify this region as a promoter region containing a subregion known as TATA box. The promoter sequence could be located using program PROSCAN Version 1.7 (24]).

The dictionary of this region provides another example of regularity in 


\begin{tabular}{|l|}
\hline AATTAAAATCAGACCGTTTCGGAATGGAAAT \\
\hline AGACCGTTTCGGAATGGAAAT \\
\hline AGACCGTTTCGGAATGGAAATGAT \\
\hline AGGGAACCCTAAAAAGGTTC \\
\hline AGGGAACCCTAAAAAGGTTCCCTTGAGGGTT \\
\hline AGGGAACCCTAAAAAGGTTCCCTTGAGGGTTCATTAAAATCAGACCGTT \\
TCGGAATGGAAATCTGTT \\
\hline AGGGAACCTAAAAAGGTTCCCTTGAGGGTTCATTAAAATCAGACCGTT \\
TCGGAATGGAAATCTGTTAGGGAACCCTAAAAAGGTTCCCTTGAGGGTT \\
CATTAAAATCAGACCGTTTCGGAATGGAAATCTGTT \\
\hline ATTAAAATCAGACCGTTTCGGAATGGAAATGATT \\
\hline CATTAAAATCAGACCGTTTCGGAATGGAAATTC \\
\hline CATTAAAATCAGACCGTTTCGGAATGGAAATCTGTT \\
\hline CCTTGAGGGTTCATTAAAATCAGACCGTTTCGGAATGGAAATCTGTT \\
\hline GTATTAAAATCAGACCGTTTCGGAAT \\
\hline GTTTCGGAATGGAAATCTGTT \\
\hline GTTTCGGAATGGAAATGAAT \\
\hline GTTTCGGAATGGAAATGATT \\
\hline GTTTCGGAATGGAAATTTTT \\
\hline TAAAATCAGACCGTTTCGGAT \\
\hline TAAAATCAGACCGTTTCGGAATGGAAAT \\
\hline TAAAATCAGACCGTTTCGGAATGGG \\
\hline
\end{tabular}

Table 6: Methanococcus jannaschii genome. Phrases longer than 20 bp are listed, coming from the dictionary relative to atypical region Inter_236189. 
DNA sequences, different from the one coming from the genome of $A r$ chaeoglobus fulgidus.

\subsection{Arabidopsis thaliana}

Arabidopsis thaliana is a small flowering plant that is widely used as a model organism in plant biology. Arabidopsis is a member of the mustard (Brassicaceae) family, which includes cultivated species such as cabbage and radish. Arabidopsis thaliana is the first plant for which the complete genome has been sequenced. Its genome consists of five chromosomes, but we have analysed only chromosomes II and IV. Since the research regarding this genome is still in itinere, here we shall present some very preliminar results concerning chromosome II.

The atypical regions we have analysed are

- Coding_8330271: atypical region, length $L=309$ bp, sublinearity in$\operatorname{dex} \mathcal{G}_{Z}=0.166$, fragment complexity $K=1.113$.

- Inter_22564763: atypical region, length $L=65849$ bp, sublinearity index $\mathcal{G}_{Z}=0.589$, fragment complexity $K=0.911$.

These regions have been chosen as peculiar among the many atypical regions (see Figure 10) belogning to this genome: a short and very regular coding region and a long intergenic region.

The atypical region Coding_8330271 is characterized by a period ' $G A$ ' that is repeated for most part of the sequence (the first $200 \mathrm{bp}$ ). This is made evident both from the $I(n)$ plot on Figure [1] $(a)$, which is definitely logarithmic in the first part, and from the word length doubling highlighted in Figure $11(b)$. Also, the multimodal distribution of word length reflects the atypical nature of this regions, while the maximal length is $12 \mathrm{bp}$, which confirms that the characteristic maximal length in non-atypical coding regions is about $11-12 \mathrm{bp}$ (for instance, see 5 (c)). The putative protein that may be obtained by translating this coding region is following protein Atg219370:

\section{ERERGSERERERERERERERERERERERERERERERER EREREREREREREREREREREREREKHKPATLAKNRRR RFVKNRRRRDHRRRISIIDGYESQF $* \mathrm{~V}$}

In the above notation, each letter corresponds to an amino acid, while the star indicates the end of the protein. This putative protein is very rich in Glutamate (E) and Arginine (R), but its function is still unknown and consideration should be given to the fact that the actual existence of this protein in the living organism has not yet been confirmed by biomolecular laboratory experiments, therefore this fragment has been classified as coding onyl by means of statistical predicitive methods . 
The atypical region Inter_22564763 was a challenging task, because not only the Information Content growth shows an abrupt change around 50000 bp (Figure $12(a)$ ), but also the word length is subjected to a deep decrease when reaching that threshold, although at that point the dictionary already contained more than 1700 phrases, most of them longer than 50 bp (Figure $12(b)$ and $(c))$.

It was this twofold look of the region that suggested that in the final part of this region (from $50000 \mathrm{bp}$ to $65849 \mathrm{bp}$ ) there might have been some coding sequences. This was also supported by the prevailing length of about $11-12 \mathrm{bp}$, which, as it was already pointed out, may be considered as characteristic of coding regions. As a result, four putative genes G1, G2, G3 and G4 have been located by means of Hidden Markov Model-based program FGENESH ${ }^{2}$ that has been created for predicting multiple genes and their structure in genomic DNA sequences. The analysis via FGENESH has been exploited with respect to known genes in Arabidopsis thaliana. Their predicted position is illustrated in Figure 13.

\section{Final remarks and future work}

We have shown that complete genomes may be analysed in some of their distinctive features by means of the Computable Information Content obtained via compression algorithms. The Information Content may be used to extract regions having an atypical information growth, which is strictly connected to the presence of highly repetitive subregions that might be supposed to have a regulatory function within the genome. Different types of sublinearities have been associated to different biogical features. These results shall pave the way for a more profound understanding of the local compressibility of genomes and for a more detailed identification of motifs and patterns that are significant to some biological function, in view of a joint use together with other predictive methods.

\section{References}

[1] Billingsley P., Ergodic Theory and Information, J. Wiley and Sons, New York (1965).

[2] Khinchin A.I., Mathematical foundations of information theory, Dover Publications, New York, 1957

[3] Chaitin G.J, Information, Randomness and incompleteness, second edition, World Scientific, Singapore (1990).

\footnotetext{
${ }^{2}$ This program is available at the website www.softberry.com to which we refer concerning the reliability and efficiency of the algorithm.
} 
[4] Kolmogorov A. N., "On the entropy per time unit as a metric invariant of automorohism", Dokl. Acad. Nauk., 124: 754-755 (1959).

[5] Bell T., Witten I. H., Cleary J. G., Modeling for text compression, ACM Computing Surveys, 21, 557-591 (1989).

[6] Benci V., Bonanno C., Galatolo S., Menconi G., Virgilio M., Dynamical systems and computable information, to appear on Disc. Cont. Dyn. Syst.- $B$.

[7] Adebiyi E. F., Jiang T., Kaufmann M.," An efficient algorithm for finding short approximate non-tandem repeats", Bioinformatics, 17, Suppl 1: S5-S12 (2001).

[8] Li M., Badger J.H., Chen X., Kwong S., Kearney P., Zhang H., "An information based sequence distance and its Application to whole mithocondrial genome phylogeny", Bioinformatics, 17 (2): 149-154 (2001).

[9] Grumbach S., Tahi F., "A new challenge for compression algorithms: genetic sequences", Information processing \& Management, 30: 875886 (1994).

[10] Galatolo S., "Orbit complexity and data compression" , Discrete and Continuous Dynamical Systems 7, 477-486 (2001).

[11] Galatolo S., "Complexity, initial data sensitivity, dimension and weak chaos in dynamical systems", Nonlinearity, 16, 4, 1219 (2003).

[12] Argenti F., Benci V., Cerrai P., Cordelli A., Galatolo S., Menconi G., "Information and dynamical systems: a concrete measurement on sporadic dynamics", Chaos, Solitons and Fractals, 13, 3, 461-469 (2002).

[13] P. Allegrini, V. Benci, P. Grigolini, P. Hamilton, M. Ignaccolo, G. Menconi, L. Palatella, G. Raffaelli, N. Scafetta, M. Virgilio, Y. Yang, "Compression and Diffusion: A Joint Approach to Detect Complexity", Chaos, Solitons \& Fractals 15, 17 (2003).

[14] Bonanno C., "The Manneville map: topological, metric and computational approach", http://arXiv.org/abs/math.DS/0107195 (2001).

[15] Fronzoni L., Galeotti L., Menconi G., "Measure of Diffusion Entropy of weak turbulence in sample of nematic liquid chrystal", in Determinism, Holism and Complexity, p.87, Atti dell'omonimo convegno tenutosi ad Arcidosso (GR), 2-8 Settembre 2001, Vieri Benci et al. editors, Kluwer Academic/Plenum Publishers, NY (2003).

[16] Bellazzini J., Menconi G., Ignaccolo M., Buresti G., Grigolini P., "Vortex Dynamics in evolutive flows: a weakly chaotic phenomenon", Physical Review E, 68: 026126 (2003). 
[17] Bonanno C., Menconi G., "Computational information for the logistic map at the chaos threshold", Disc. Cont. Dyn. Syst.- B, 2, no.3, 415431 (2002).

[18] Ziv J., Lempel A., "A Universal Algorithm for Sequential Data Compression", IEEE Transactions on Information Theory, 23, 337-342 (1977).

[19] Ziv J., Lempel A., "Compression of Individual Sequences Via VariableRate Coding", IEEE Transactions on Information Theory, 24, 530-536 (1978).

[20] Bell T., Witten I. H., Cleary J. G., Modeling for text compression, ACM Computing Surveys, 21, 557-591 (1989).

[21] Benedetto D, Caglioti E., Loreto V.,"Language trees and zipping", Phys Rev Lett 88(4):048702(2002).

[22] Myers G., "Whole-genome DNA sequencing", Computing in Science 8 Engineering, 1, 3:33-43 (1999).

[23] Cover T. M., Thomas J. A., Elements of Information Theory, Wiley (1991).

[24] Prestridge, D.S., "Predicting Pol II Promoter Sequences Using Transcription Factor Binding Sites", J. Mol. Biol.,249: 923-32 (1995). 
(a)

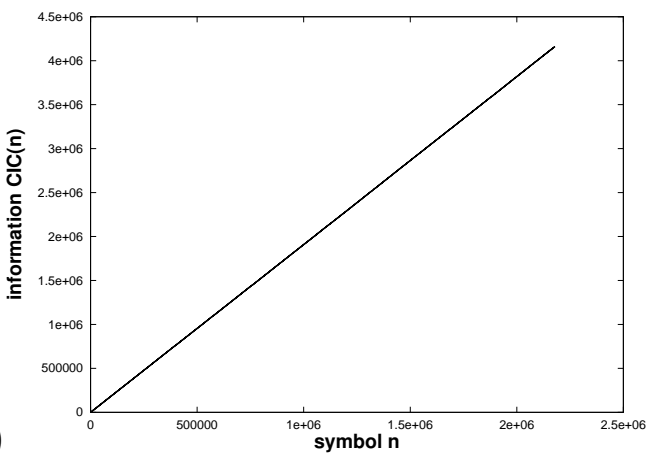

(b)

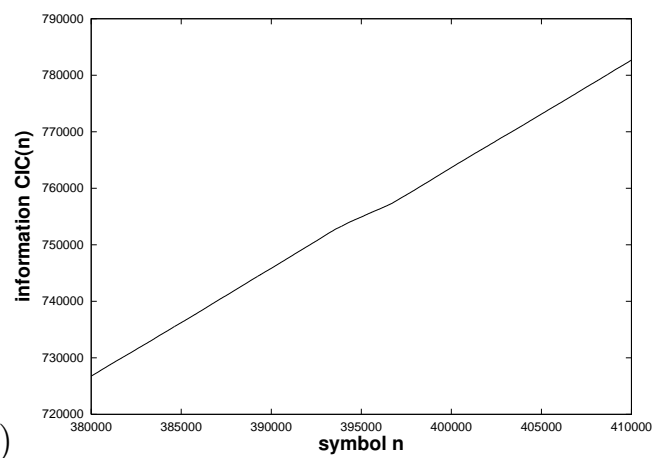

Figure 1: (a) complete $C I C(n)$ graph for Archaeoglobus fulgidus complete genome; (b) local enhancement of the region from 380000 to $410000 \mathrm{bp}$. The behaviour of $C I C(n)$ is no more linear.

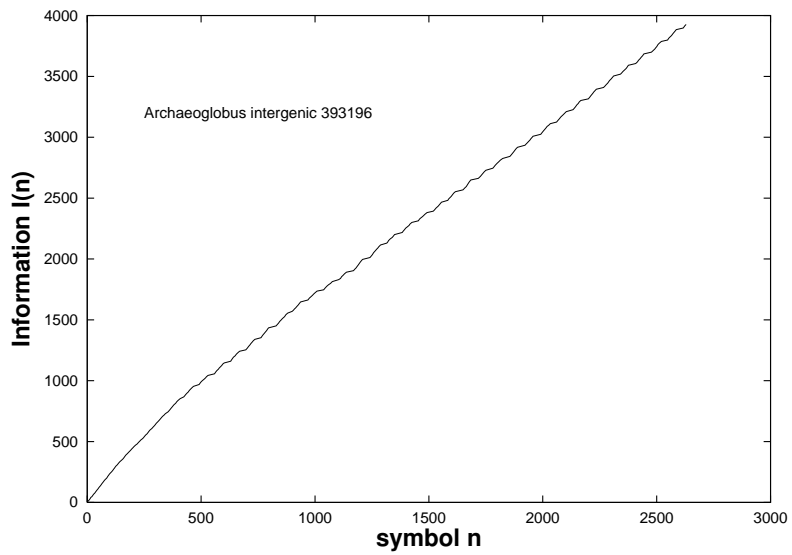

Figure 2: Archaeoglobus fulgidus genome. The behaviour of the information content of region Inter_393196 is a power law whose exponent is 0.832. The picture is in linear scale. 


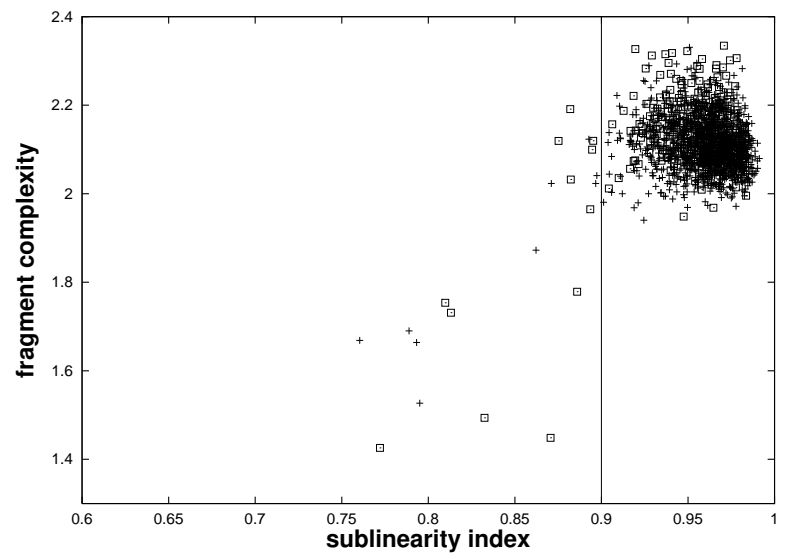

Figure 3: Archaeoglobus fulgidus genome. Comparison between the values of sublinearity index and fragment complexity of all functional regions with length greater than $200 \mathrm{bp}$. The crosses (+) are referred to coding regions, while the diamonds $(\diamond)$ are referred to intergenic regions. The vertical line is the threshold for the sublinearity index, under which the region is atypical.

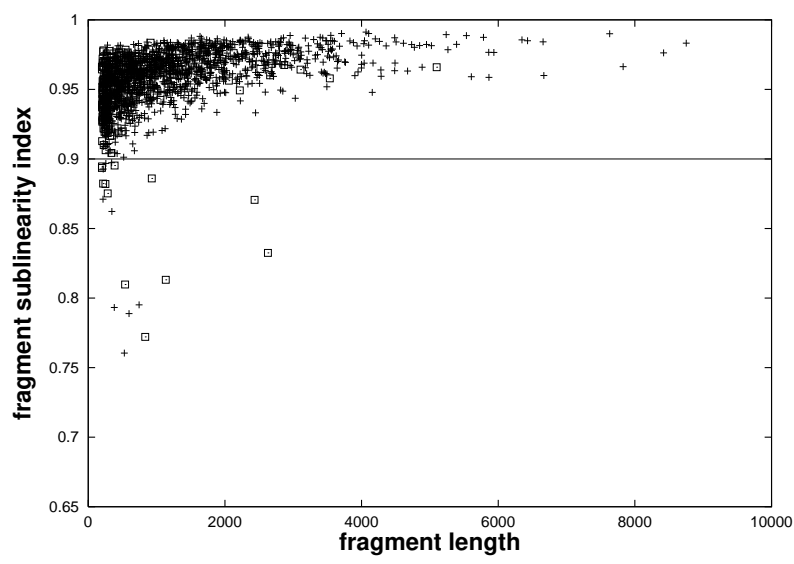

Figure 4: Archaeoglobus fulgidus genome. Of each functional region, its length and the corresponding sublinearity index are plotted. The crosses (+) are referred to coding regions, while the squares $(\square)$ are referred to intergenic regions. The horizontal line is the threshold for the sublinearity index, under which the region is atypical. 
(a)

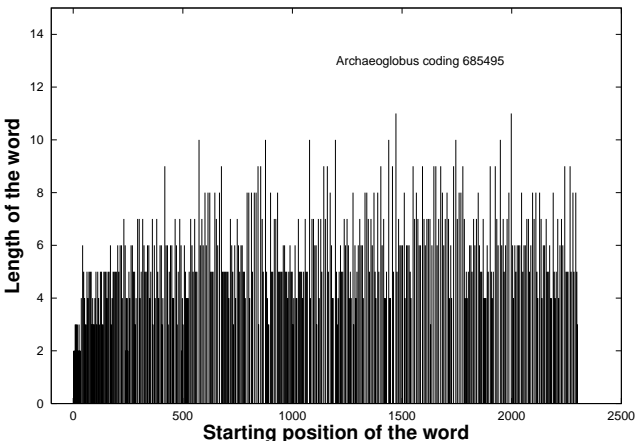

(c)

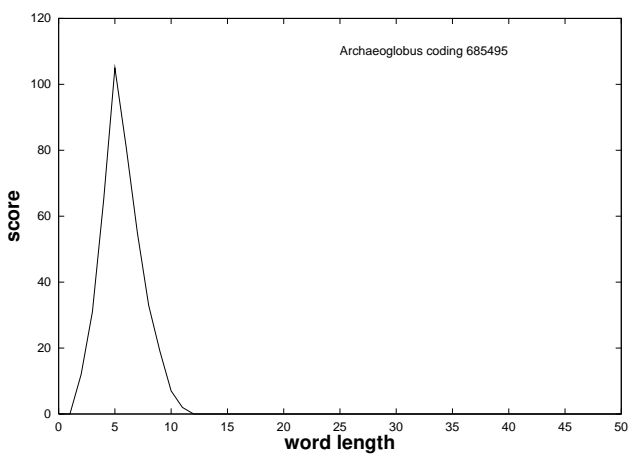

(b)

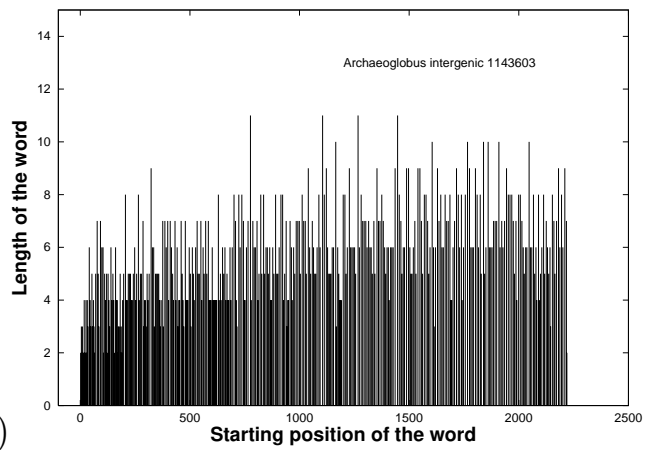

(d)

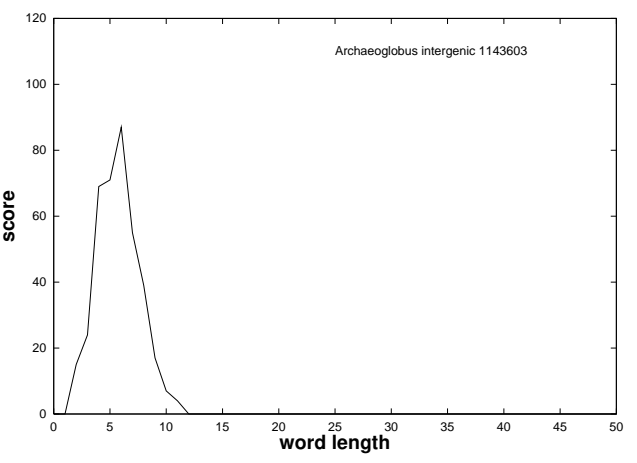

Figure 5: Archaeoglobus fulgidus genome. Plots (a) and (b) show the location and length of the phrases in the parsing by the algorithm CASToRe, in nonatypical regions Coding_685495 and Inter_1143603, respectively. Graphs (c) and (d) illustrate the distribution of phrase length in the same regions.

(a)

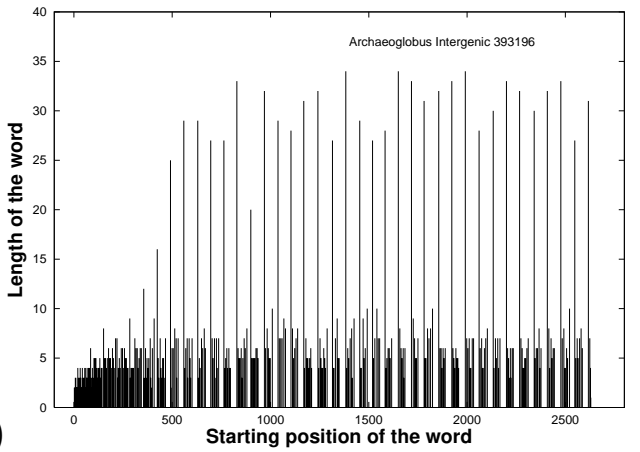

(b)

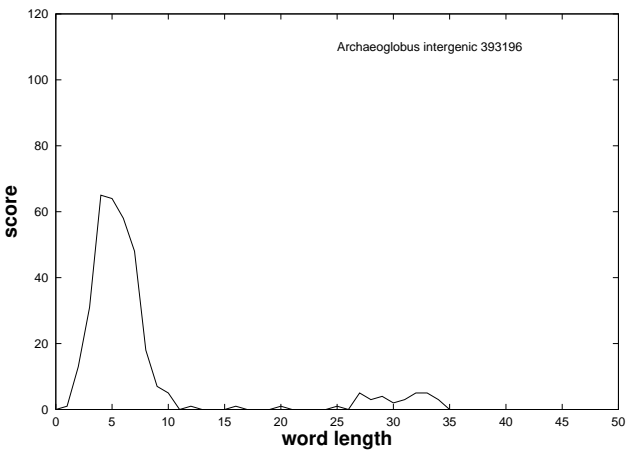

Figure 6: Archaeoglobus fulgidus genome. Plot (a) shows the location and length of the phrases in the parsing by the algorithm CASToRe, in atypical region Inter_393196. Graph (b) illustrates the distribution of phrase length in the same region. 


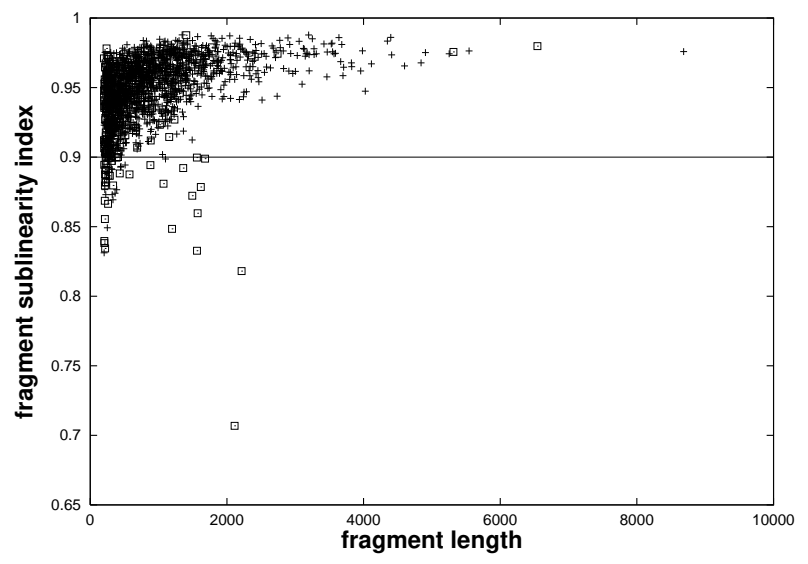

Figure 7: Methanococcus jannaschii genome. Of each functional region, its length and the corresponding sublinearity index are plotted. The crosses (+) are referred to coding regions, while the squares $(\square)$ are referred to intergenic regions. The horizontal line is the threshold for the sublinearity index, under which the region is atypical.

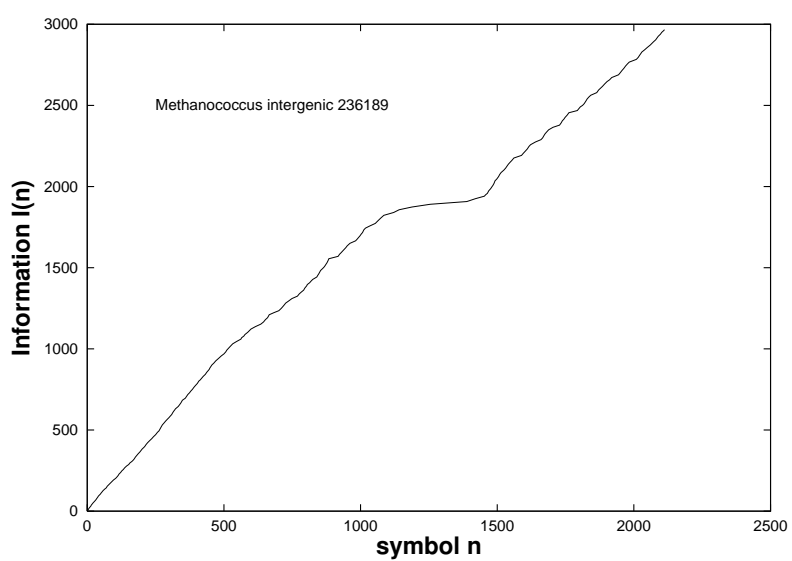

Figure 8: Methanococcus jannaschii genome. The behaviour of the information content of region Inter_236189 grows sublinearly with index 0.70\%. The picture is in linear scale. 


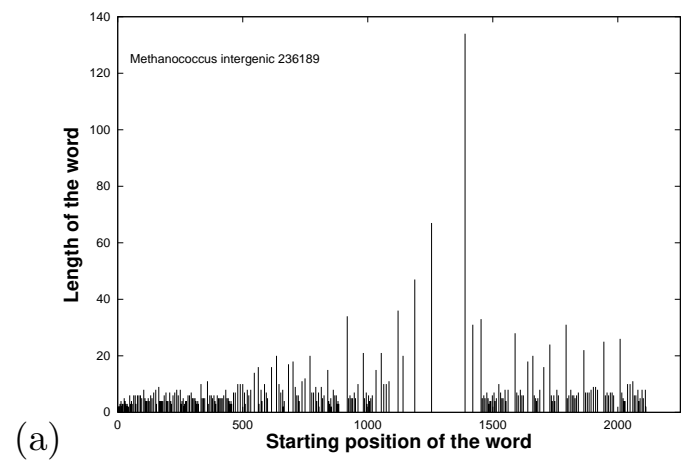

(b)

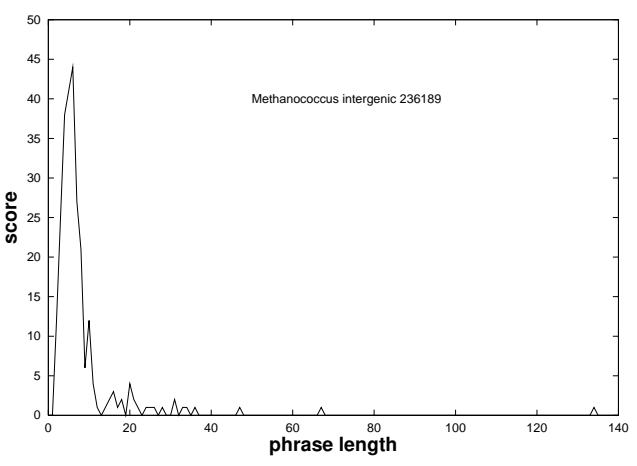

Figure 9: Methanococcus jannaschii genome. Plot $(a)$ shows location and length of the phrases in the parsing by the algorithm CASToRe of region Inter_236189. In graph (b) the corresponding distribution of phrase length is pictured.

(a)

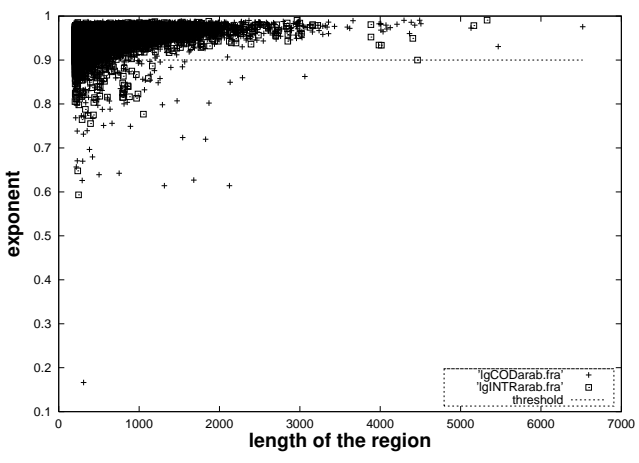

(b)

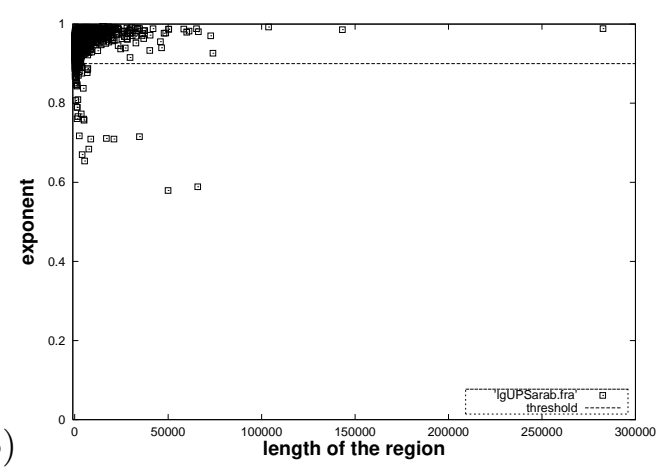

Figure 10: Arabidopsis thaliana genome. Of each functional region, its length and the corresponding sublinearity index are plotted. In picture (a), the crosses (+) are referred to coding regions, while the squares ( $\square)$ are referred to introns. In picture (b), the squares $(\square)$ are referred to intergenic regions. In both plots, the horizontal line is the threshold for the sublinearity index, under which the region is atypical. 
(a)

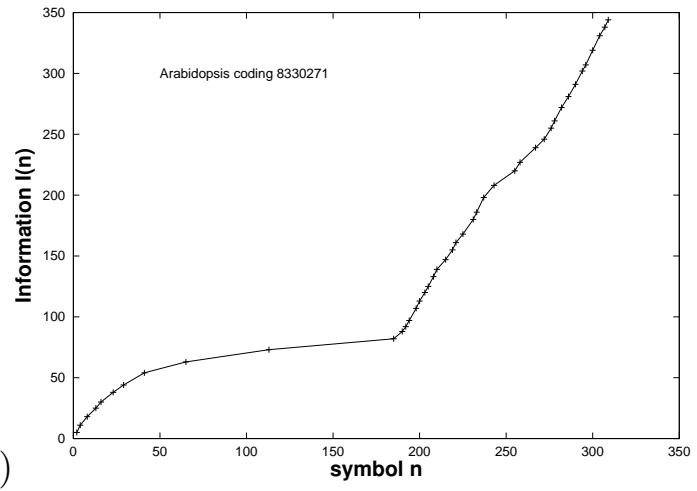

(b)

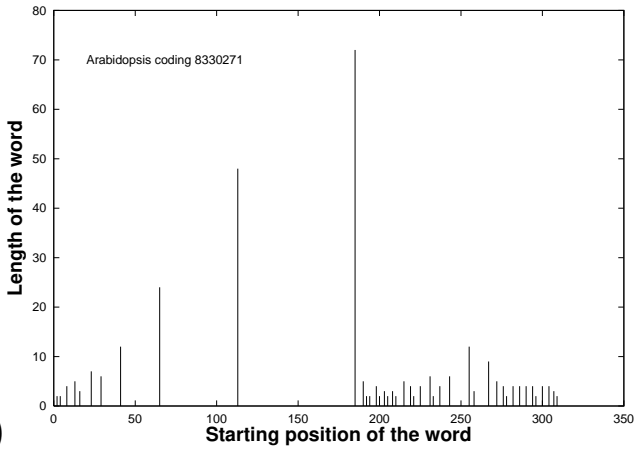

(c)

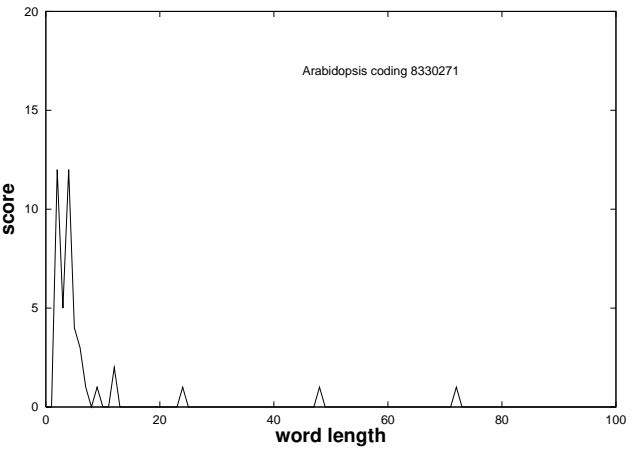

Figure 11: Atypical region Coding_8330271. (a) The Information Content growth is logarithmic for the main part of the sequence. The word length doubling is shown on plot (b) and the multimodal distribution of word length is illustrated in (c). 


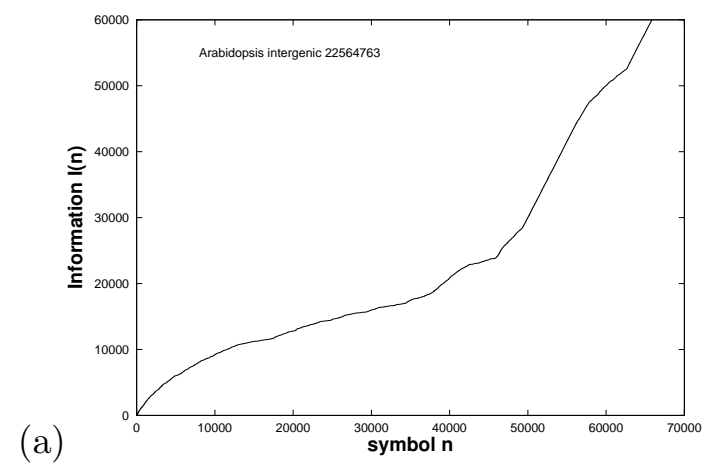

(b)

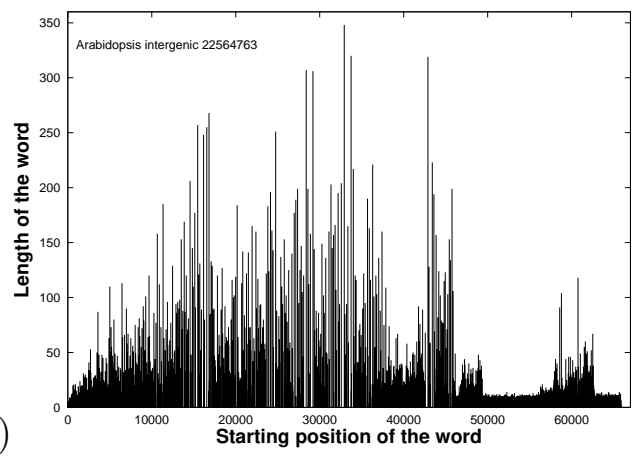

(c)

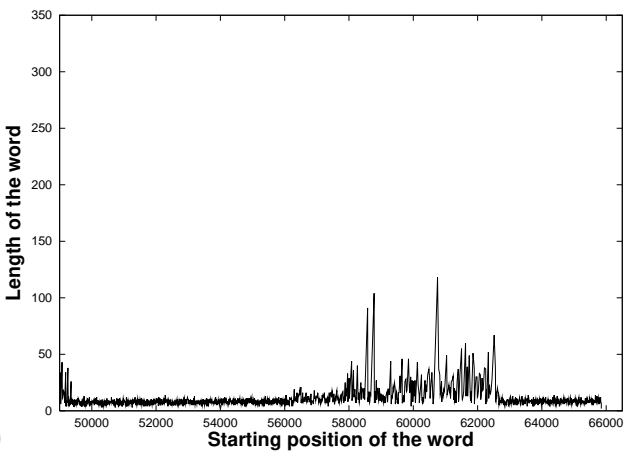

(d)

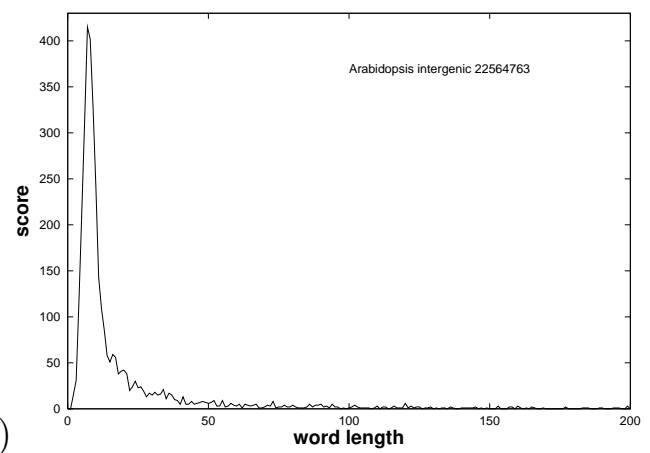

Figure 12: Arabidopsis thaliana genome (chromosome II). (a) The behaviour of Information Content of atypical region Inter_22564763 grows in a very peculiar way. Its sublinearity index has been evaluated as 0.589. (b) The plot shows location and length of the phrases in the parsing obtained by the algorithm CASToRe. (c) The plot is an enhancement of the final part of the atypical region Inter_22564763. (d) The distribution of phrase length for the aforementioned parsing is pictured. 


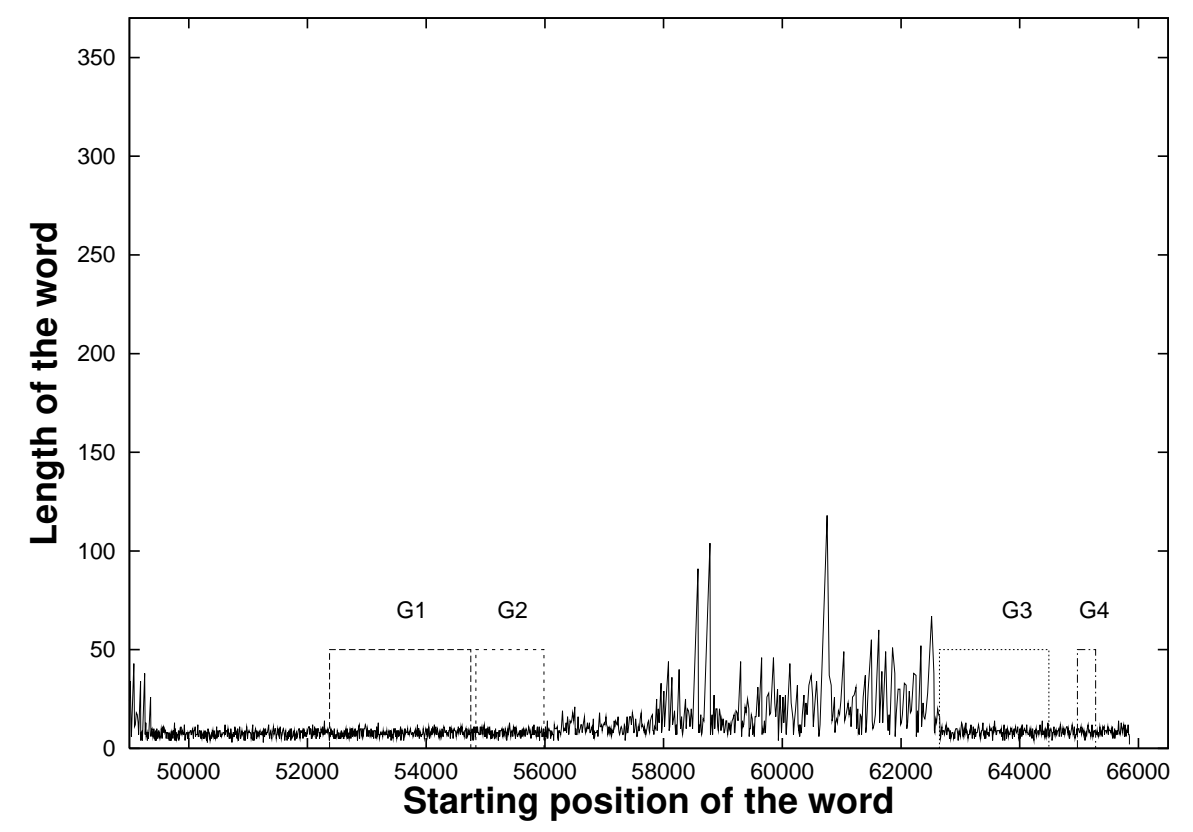

Figure 13: Arabidopsis thaliana genome (chromosome II). Same part of atypical region Inter_22564763 as plot (c) in Figure 12. The boxes correspond to the location of the four predicted genes (labelled as

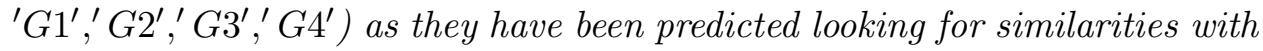
Arabidopsis thaliana known genes. 